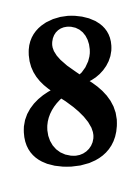

\title{
EL PROYECTO EUROPEO ECO. ROMPIENDO BARRERAS EN EL ACCESO AL CONOCIMIENTO
}

\author{
(THE EUROPEAN PROJECT ECO. BREAKING DOWN \\ BARRIERS TO ACCESS KNOWLEDGE)
}

Sara Osuna-Acedo

Javier Gil-Quintana

UNED

DOI: $10.5944 / e d u c X X 1.19037$

\section{Cómo referenciar este artículo/How to reference this article:}

Osuna-Acedo, S. y Gil-Quintana, J. (2017). El proyecto europeo ECO. Rompiendo barreras en el acceso al conocimiento. Educación XX1, 20(2), 189-213, doi: 10.5944/educXX1.19037

Osuna-Acedo, S. \& Gil-Quintana, J. (2017). El proyecto europeo ECO. Rompiendo barreras en el acceso al conocimiento. [The european project ECO. Breaking down barriers to access knowledge]. Educación XX1, 20(2), 189-213, doi: 10.5944/educXX1.19037

\section{RESUMEN}

El Proyecto europeo ECO (E-learning, Communication, Open-Data) busca desarrollar un modelo educativo horizontal y bidireccional, desde la nueva realidad de los MOOC. Con base en los principios de equidad, inclusión social, accesibilidad, autonomía y apertura, el empoderamiento del alumnado se hace realidad con esta propuesta de formación, rompiendo las barreras del escenario digital del curso para repercutir, desde las redes sociales, en la capa social. Tomando como referente el impulso formativo de los MOOC de ECO, los resultados que se presentan en este estudio han sido analizados desde una metodología cuantitativa optando por el análisis, como muestra intencional, de los datos obtenidos en la primera convocatoria de cursos. Se ha optado por este tipo de análisis para identificar los rasgos que han caracterizado a este modelo formativo, la demanda que ha obtenido y su desarrollo a nivel pedagógico. Como podremos comprobar, este estudio ha dejado patente que ECO ha potenciado de forma masiva los MOOC haciéndose presente, no solo en el ámbito europeo, sino también en otros países, especialmente en aquellos que están en vías de desarrollo. Esta oferta formativa se ha implementado con éxito en el ámbito docente con la finalidad de fomentar el descubrimiento de recursos abiertos y estrategias para la mejora de la calidad educativa, ofreciendo la posibilidad de crear sus propios cursos 
como medio para compartir sus conocimientos con otros docentes. Como consecuencia, el éxito de los MOOC y la publicitación de los mismos vienen determinados por la experiencia, la conformidad con los contenidos y por el grado de satisfacción del alumnado participante, convirtiéndose en publicista de los mismos en sus diferentes espacios sociales. El Proyecto europeo ECO se presenta como modelo de formación accesible que permite, no solo el diseño universal, sino también la apertura y la participación en esta formación para toda la ciudadanía.

\section{PALABRAS CLAVE}

Educación innovadora; tecnología aplicada a la educación; educación mediática; educación abierta.

\section{ABSTRACT}

The European Project ECO (E-learning, Communication, Open-Data) seeks to develop an educational model that is horizontal and bidirectional, portraying the new reality of the MOOC. On the basis of the principles of equity, social inclusion, accessibility, autonomy and openness, the empowerment of the students becomes real with this training proposal, breaking down the barriers of the digital scenario of the course in order to have an impact, through social media, on the social layer. Taking the educational momentum of the ECO MOOC's, the results presented in this study have been analyzed using a quantitative methodology using the analysis of an intentional sample with the data obtained in the first round of courses. This type of analysis aims to identify the traits that have characterized this educational model and the demand that has been demonstrated with development on a pedagogical level. As we can see, this study has made it clear that ECO has massively increased the presence of MOOC's, not only on a European level but also in other countries, even in those that are in the process of development. This training has been implemented successfully within the teaching sphere with the clear objective of encouraging the discovery of open resources and strategies for the improvement of educational quality, offering the possibility of creating their own courses as a means to share knowledge with other teachers. As a result, the success of the MOOC and publicizing of the same are determined by the experience, the conformity with the contents and the degree of satisfaction of the students participating, becoming a publicist of the same in its different social spaces. The European Project ECO is presented as a model of accessible learning that allows not only a universal design, but also its openness and the participation in this education for all citizens.

\section{KEYWORDS}

Innovative education; technology applied to education; media education; open education. 


\section{INTRODUCCIÓN}

Los MOOC se han presentado como una revolución con un poderoso potencial de cambio en los contextos de formación. Se trata de cursos gratuitos ofrecidos a gran escala por instituciones educativas, con carácter masivo, porque pueden ser cursados por miles de personas de forma online en un mismo momento y desde cualquier parte del mundo y, finalmente, abiertos porque las instituciones que los ofertan proporcionan al alumnado recursos educativos en abierto permitiendo «complementar acciones dirigidas a la internacionalización del mismo» (Sancho Vinuesa, Oliver \& Gisbert, 2015). Estos cursos se asientan sobre distintos escenarios digitales que toman diferentes matices, según el criterio metodológico por el que se haya apostado, y se basan en «el aprendizaje distribuido en red, fundamentado en la teoría conectivista y su modelo de aprendizaje; las tareas, según las habilidades del alumnado en la resolución de determinados tipos de trabajo y los contenidos» (Raposo, Martínez-Figuera \& Sarmiento, 2014, p. 2).

Este trabajo parte del análisis de una propuesta de cursos masivos, abiertos y online que se ha realizado en veintiuna instituciones europeas encabezadas por la Universidad Nacional de Educación a Distancia (UNED) a través del Proyecto europeo ECO (E-learning, Communication, Open-Data). Partiendo de un marco teórico y de un fundamento metodológico y comunicativo, se ha abordado la investigación sobre la proyección formativa de ECO a nivel internacional, analizando el perfil académico de los destinatarios de estos cursos y estudiando la satisfacción de los mismos ante la formación recibida. El contenido, que de forma específica y en base a unas hipótesis concretas se ha analizado, ha estado fundamentado en el análisis de los resultados que se han manifestado en los cuestionarios finales que, de forma anónima, han sido cumplimentados por doscientos ochenta y nueve participantes en los diferentes MOOC que la plataforma del Proyecto ECO ofrecía en su primera convocatoria.

\section{Los MOOC, un nuevo modelo formativo desde la universidad}

Los usuarios de Internet estamos siendo testigos de esta nueva ola que da paso a la democratización del acceso al conocimiento, para que la ciudadanía pueda «alcanzar el objetivo de una educación abierta para todos» (Román Mendoza, 2014, p. 35). Las instituciones educativas han abierto las puertas de sus aulas para compartir con la sociedad, a nivel internacional, gran parte del conocimiento desarrollado por sus profesionales, haciendo "que cada individuo pueda ahora convertirse en un nodo de comunicación que da y recibe simultáneamente» (Sangrá \& Wheeler, 2013, p. 107). 
En el video de Cormier (2010) ¿Qué es un MOOC? de la red social Youtube se ofrece una aclaración concreta sobre este concepto. Actualmente, los cursos MOOC se han convertido en una nueva oportunidad para aprender en base a una comunidad de practica o de aprendizaje, constituyen una alternativa cada vez más presente en la sociedad en que vivimos y son resultado, precisamente, del potencial de Internet en el ámbito de la comunicación y la interacción de los usuarios, así como de la enorme facilidad de actualización del conocimiento que se permite conseguir. El cronograma de estudio y la obligatoriedad de cursar todos los contenidos de este tipo de cursos es libre, el alumnado podrá empezar o terminar los mismos cuando lo desee y además algunos MOOC tienen la posibilidad de solicitar una certificación donde se especifican los créditos que contienen. En este sentido, Siemens et al. (2012) señalan:

No espere poder leer ni ver todo. Incluso nosotros, profesores, no podemos hacerlo. En cambio, lo que se debe hacer es seleccionar y elegir el contenido que vea interesante y adecuado para usted. Si le parece demasiado complicado, no lo lea. Si lo ve aburrido, pase al siguiente. Los resultados del aprendizaje, por lo tanto, serán diferentes para cada persona. (Siemens, et al., 2012).

Actualmente, la aparición de un gran número de propuestas que se alejan de la concepción primaria de los MOOC, ha hecho que esta definición original se tenga que replantear, debido a que esta noción es tan amplia y ambigua que incluso hay discusiones sobre si los MOOC son realmente un curso o son "una especie de texto docente mejorado» (Pernías \& Luján, 2014). Se pueden considerar los MOOC como

«un progreso de tendencia tecnológica y social, especialmente en el panorama del estudio superior para la estimulación orientada a la innovación y promoción del aprendizaje en masa, abierta e interactivamente, es decir, la génesis de la investigación colectiva» (Tobías-Martínez, Duarte-Freitas y Kemczinski, 2015, p. 64).

En este planteamiento podemos distinguir varios tipos de MOOC con diferentes propuestas de interacción y comunicación entre los participantes de la comunidad de práctica o aprendizaje, con disimilitud entre los objetivos, las metodologías y los diferentes resultados que se esperan. En consecuencia, diferenciamos principalmente tres tipos: xMOOC cursos basados en modelos comunicativos conductistas, que surgen con la finalidad de reproducir el carácter instrumental de la tecnología en el aula a los modelos de enseñanza masiva; cMOOC cursos claramente conectivistas por su finalidad para construir el conocimiento a través de un modelo de comunicación que favorece el intercambio y el aprendizaje interconectado; y sMOOC como propuestas que se apropian del canal social media provocando una ruptura con el sistema 
establecido y enriqueciendo la capa social con un conocimiento compartido, que se identifican con las relaciones interpersonales desarrolladas entre los miembros de estos entornos virtuales. En palabras de Gil-Qintana (2015):

El éxito activista de estos proyectos se fundamenta en una participación interactiva que se dirige más allá de las barreras de la plataforma del curso, de forma habitual, a través de social media, característica intrínseca a la pedagogía conectivista. El aprendizaje se difunde por todos los espacios del software social que contribuyen a la edificación de la inteligencia colectiva. (Gil-Quintana, 2015, p. 304).

El desarrollo de Internet ha otorgado el protagonismo a los usuarios uniéndose «las posibilidades de la Web 2.0 con los contenidos abiertos para un aprendizaje permanente de forma social y colaborativa, como los denominados MOOC» (Rodríguez Terceño, 2012, p.246). Las iniciativas que se han desarrollado desde la implantación de esta versión de formación en la Red han triunfado como medio para dar la voz a la ciudadanía, dándole opciones de aportar, colaborar, interactuar, etc., estructurando, de esta forma, un conocimiento compartido. Cantillo define estas prácticas como «ecosistemas de conectivismo» donde se plantea «una pedagogía en la que el conocimiento surge a partir de las relaciones y debates que se establecen entre los miembros de estas comunidades; por tanto, el aprendizaje se concibe como una actividad continua y permanente» (Cantillo 2014, p.59). De esta forma, una de las motivaciones que han llevado a la ciudadanía a apropiarse de la Red ha sido la necesidad de satisfacer sus demandas de aprendizaje. Por este motivo, han inundado las diferentes plataformas de tutoriales y manuales que presenta una especie de solidaridad digital compartiendo con los demás lo que es propio, resolviendo conjuntamente problemas y aprendiendo de forma colaborativa. Esta elección del alumnado obedece, sin duda alguna, a que cada vez más se busca escoger lo que interesa o se necesita en términos de formación, por encima de la necesidad de almacenar credenciales que decoran nuestro currículum vitae, pero no condicionan en positivo nuestra valía a nivel académico y profesional. Este hecho nos puede deparar a un futuro muy próximo en que las universidades del siglo XXI, vayan «perdiendo - paulatinamente, como ya lo vienen haciendo- el liderazgo académico en términos de conocimiento válido para la sociedad en red» (Luque, 2015, p. 1).

\section{El proyecto europeo ECO}

En el año 2014, distintas instituciones, entre las que destaca en España la Universidad Nacional de Educación a Distancia (UNED), se han unido con la finalidad de apostar por esta innovación a nivel mundial a través del Proyecto europeo ECO. Se han tomado como base las tecnologías 
de vanguardia para implementar metodologías educativas que, a través del software social, activan mecanismos de participación y comunicación colectiva, repercutiendo masivamente en la difusión del conocimiento. ECO otorga, por tanto, a la nueva formación online un enfoque educomunicativo, "prestando atención a estas nuevas formas de aprender a través de la Web y estas nuevas teorías del aprendizaje» (Barbas, 2012, p. 172). Como señalan Osuna-Acedo y Aparici (2014):

Los Entornos Virtuales Educomunicativos han de distinguirse por provocar actitudes críticas y reflexivas sobre la construcción del conocimiento, donde la interactividad y la flexibilidad generan una comunicación entre sus miembros, que les permita reconocerse como sujetos activos en el proceso de enseñanza-aprendizaje. Todos los sujetos implicados pueden intercambiar roles de emisores y receptores para alcanzar un verdadero aprendizaje significativo. ( $p .14)$.

ECO desde el «Programa marco para la competitividad y la innovación (CIP)» está contribuyendo, por medio del acceso abierto a la formación por parte de la ciudadanía, a la construcción colectiva del conocimiento desde Europa. Este planteamiento educacional pretende huir de todo tipo de comunicación jerárquica y unidireccional, abriendo paso a un «espacio complejo de múltiples entradas, recorridos y salidas interrelacionadas y en movimiento» (Silva, 2005, p.47). El proyecto ha demostrado el potencial de los MOOC para la destrucción de todo tipo de barreras tecnológicas que han provocado, desde tiempos pasados, la exclusión del conocimiento a sectores sociales determinados. Este impacto de ECO se ha visto incrementado por la preparación e implicación del equipo de docentes y de facilitadores, que ha sido capaz de crear sus propios cursos online, difundiéndolos mediante la plataforma de aprendizaje que recoge todo el proyecto formativo. Como se indica en la web del proyecto project.ecolearning.eu, referido a los MOOC:

LOS MOOC pueden aplicarse al aprendizaje formal/informal/no formal y hacer que el aprendizaje sea ubicuo. ECO pondrá su enfoque en extender a escala paneuropea las experiencias MOOC más exitosas de Europa, pilotando y mostrando las mejores prácticas a través de su implementación en centros regionales de excelencia en toda Europa, evaluando los desenlaces, resultados y lecciones aprendidos de estas mejores prácticas en el aprendizaje de acceso abierto y móvil.

Este proyecto se asienta sobre una serie de objetivos que se planifican a largo plazo, con una perspectiva de tres años y concretados, tal y como consta en su documento de trabajo, en: 
- Analizar los requisitos para plataformas MOOC desde un punto de vista pedagógico.

- Establecer un marco para el diseño e implementación de cursos sMOOC.

- Diseñar la arquitectura general de la plataforma ECO e integrar todos los módulos individuales en la plataforma global.

- Pilotar con la plataforma ECO en los diez centros que participan.

- Analizar y evaluar la situación del mercado internacional MOOC.

- Desarrollar un plan de estrategia de negocios apropiado para la sostenibilidad de ECO.

- Aumentar la conciencia en Europa sobre los beneficios de recursos educativos abiertos para los ciudadanos y las instituciones.

La clave que busca este proyecto es apostar por la propuesta sMOOC cuya finalidad es el empoderamiento de los estudiantes. Se les posibilita una plataforma donde por sí mismos pueden crear su propio MOOC después de recibir una formación clave para ello en el curso $s M O O C$ Paso a Paso. Se presentan por tanto los $\mathrm{SMOC}$, como proceso único de aprendizaje que tiene impacto social desde la comunidad formada en su entorno:

Los cursos han de estimular el conectivismo, el aprendizaje socioconstructivista y el aprendizaje situado. Han de dirigirse hacia el desarrollo del aprendizaje autónomo y estar centrados en el participante. El diseño ha de orientarse a crear oportunidades para el aprendizaje colaborativo y buscar estrategias o recursos para facilitar un aprendizaje adaptativo. (sMOOC Paso a Paso, 2014-17. Proyecto ECO).

El Proyecto ECO debido a que ha propuesto una colección de cursos, brinda la posibilidad de una participación activa por un gran número de estudiantes de distintas nacionalidades. Con este planteamiento de formación se proporciona un espacio para el crecimiento de la ciudadanía en la creación de nuevas temáticas y entornos de aprendizaje, propiciando, como señala Freire (2002, p. 151) «que el saber tiene todo que ver con el crecer es un hecho. Pero es necesario, absolutamente necesario, que el saber de las minorías dominantes no prohíba, no asfixie, no castre el crecer de las inmensas mayorías dominadas». 
En este sentido, la muestra intencional de nuestro análisis son los cursos presentados en la plataforma del Proyecto ECO que se han ofertado durante el curso 2014-2015. Según el proceso de análisis, se han estudiado sus MOOC como un objeto claramente delimitado, aunque han sido los responsables de este trabajo quienes han fijado las fronteras en último término. Se pretende un acercamiento a la realidad de la formación masiva, online y abierta. En el proceso realizado confluyen gran variedad de fuentes que pueden ayudarnos a descubrir el significado de las hipótesis para poder construir conclusiones que contribuyan a la creación de teorías. Como investigadores, no solo hemos pretendido un conocimiento científico del objeto de estudio en base a unos objetivos, sino que hemos llevado a cabo una práctica social debidamente sustentada para resolverlos. En consecuencia, teniendo presentes las indicaciones anteriormente citadas, se han pretendido los siguientes objetivos:

- Descubrir la proyección del Proyecto ECO en el mundo.

- Analizar el perfil académico y profesional del alumnado de MOOC del Proyecto ECO.

- Identificar los espacios donde se ha obtenido información sobre el Proyecto ECO.

- Valorar la opinión de los participantes en la primera edición de los cursos del Proyecto ECO en relación a su satisfacción personal.

\section{MÉTODO}

El estudio que presentamos a continuación se ha basado en el método científico, siguiendo unos pasos lógicos y sistemáticos que han posibilitado la comprobación veraz de nuestras conclusiones. Para afirmar la autenticidad de este proceso ha sido imprescindible seguir un método concreto, considerado por la comunidad científica como el más apropiado para analizar esa realidad concreta. En palabras de Coller (2005):

El método científico [...] requiere el acuerdo implícito de la comunidad cientifica acerca de las reglas que gobiernan el proceso de comprobación de una hipótesis que se refiere al funcionamiento de la sociedad o de un fenómeno particular. En términos generales, tales hipótesis establecen la relación (habitualmente causal) que existe entre dos (o más) fenómenos, variables y observaciones. (Coller, 2005, p.18). 
El estudio de caso, elegido como metodología investigativa, ha sido objeto de análisis durante décadas por muchos investigadores que han buscado la combinación de métodos de naturaleza diferente, fomentando una imaginación sociológica para poder encontrar la forma más adecuada de producir mejor el conocimiento sobre el hecho a analizar. Nuestro estudio de caso elegido es el Proyecto ECO, que se ha enfrentado a la realidad mediante un diagnóstico detallado de los elementos y la interacción que se produce entre los mismos y su contexto, llegando, mediante un proceso de síntesis, a la búsqueda del significado y a la toma de decisiones. Definidas las piezas importantes del proceso de investigación en lo referente al tema específico, justificado, englobado y delimitado en un planteamiento real, que busca analizar y resolver a través de los objetivos indicados anteriormente, llegamos al razonamiento de que «la investigación científica, en su sentido más amplio presenta dos vertientes distintas: el descubrimiento de los hechos y la creación de hipótesis y teorías» (Alcina, 1999, p. 71). De ahí que el siguiente paso para el encauzamiento del proceso sea la formulación de las hipótesis que presentamos a continuación:

- La proyección formativa del Proyecto ECO es internacional, siendo a través de Internet donde los participantes tuvieron conocimiento de sus cursos.

- Los participantes en los MOOC de ECO tienen formación universitaria al estar este Proyecto enfocado a los docentes.

- El nivel de satisfacción de los sujetos participantes por la formación recibida es alto.

En nuestro estudio, y tal como indicábamos previamente, el análisis se ha delimitado a los datos cuantitativos recogidos a través de un cuestionario digital, individual y anónimo cumplimentado por doscientos ochenta y nueve participantes en la primera edición de los diferentes MOOC de ECO, que han estado matriculados en los cursos del proyecto desde octubre de 2014 a enero de 2015. El cuestionario se ofrecía al final de cada MOOC para garantizar la recogida de datos de quienes hubieran realizado todo el proceso de aprendizaje en el MOOC.

\section{RESULTADOS}

En función de los objetivos e hipótesis especificados, el análisis de los resultados presenta los datos con lo que hemos trabajado en este estudio y de los que hemos extraído las conclusiones del mismo. Primeramente, analizamos el alcance del proyecto ECO y el perfil académico y profesional 
de los estudiantes de sus MOOC, que nos permite conocer el contexto en el que nos movemos para comprobar el grado de satisfacción que obtienen sus participantes. Para esto se han formulado los siguientes códigos por categorías que se van a contabilizar en el estudio con el fin de clasificar las respuestas de la muestra:

- Análisis de la procedencia de los estudiantes.

- Permite conseguir una interactividad intercultural en el curso

- Estimula la participación a nivel planetario

- Valoración del alcance de la diseminación del proyecto:

- Alcance en entornos analógicos

- Alcance en entornos virtuales

- Clasificación de los perfiles académicos y profesionales de los participantes en los MOOC del Proyecto ECO:

- Análisis del nivel de estudios previos

- Análisis del área profesional de procedencia

- Satisfacción por la participación en los MOOC del Proyecto ECO:

- Cumplimiento de los objetivos del MOOC

- Intencionalidad de terminar el curso en otras convocatorias

- Satisfacción sobre los materiales audiovisuales del curso

- Satisfacción sobre los planteamientos de los trabajos solicitados en el curso

- Intencionalidad de hacer más MOOC en el Proyecto ECO

- Satisfacción acerca de la usabilidad de la plataforma virtual de curso

- Contenidos accesibles para personas con distintas habilidades de aprendizaje

La masividad, característica propia de los MOOC (Martin, 2012; Vázquez-Cano, López-Meneses \& Sarasola, 2013; Yuan \& Powell, 2013; Downes, 2013; López-Meneses, Vázquez-Cano \& Román, 2014; Osuna-Acedo, 2014), se ha hecho muy presente en las propuestas del Proyecto ECO. Como hemos podido corroborar en base a la primera hipótesis de nuestro estudio, la oferta formativa de ECO se ha extendido a múltiples nacionalidades, entre las que destacan España $(44,59 \%)$, Portugal $(20,27 \%)$, Italia $(8,11 \%)$ y Francia $(5,74 \%)$. Estos países son los que sobresalen, como podemos visualizar en el Gráfico 1, pero también están presentes más abajo países del tercer mundo, 
como Zimbawe $(0,34 \%)$ y Mozambique $(0,34 \%)$, donde se forma a través de MOOC al personal docente que se enfrenta diariamente con realidades en las que se encuentra tan presente el «oprimido» (Freire, 1975) de la sociedad. En este sentido nuestro análisis coincide con propuestas ya realizadas para desarrollar el potencial MOOC en la inclusión social. Es el caso del doctor sirio Mahmud Angrinia, citado por Curley, quien detallaba la importancia de este modelo formativo masivo en entornos desfavorecidos, como los conflictos bélicos o campos de refugiados, y alienta a su alumnado a que se animen a seguir contribuyendo al proceso de reconstrucción de sus países, aprendiendo nuevas destrezas, que solo es posible mediante un "proceso formativo continuo» a través de MOOC (Curley, 2014). Asimismo, UNESCO y Commonwealth of Learning recomiendan a los países en desarrollo la necesidad de aprovechar el potencial de los MOOC para la educación online con el fin de conseguir una apertura que no solo implicaría eliminar barreras de acceso a la educación y a las tecnologías, «sino que también tiene que ver con los desafíos culturales, sociales e institucionales inherentes» (Patru \& Balaji, 2016, p. 34).

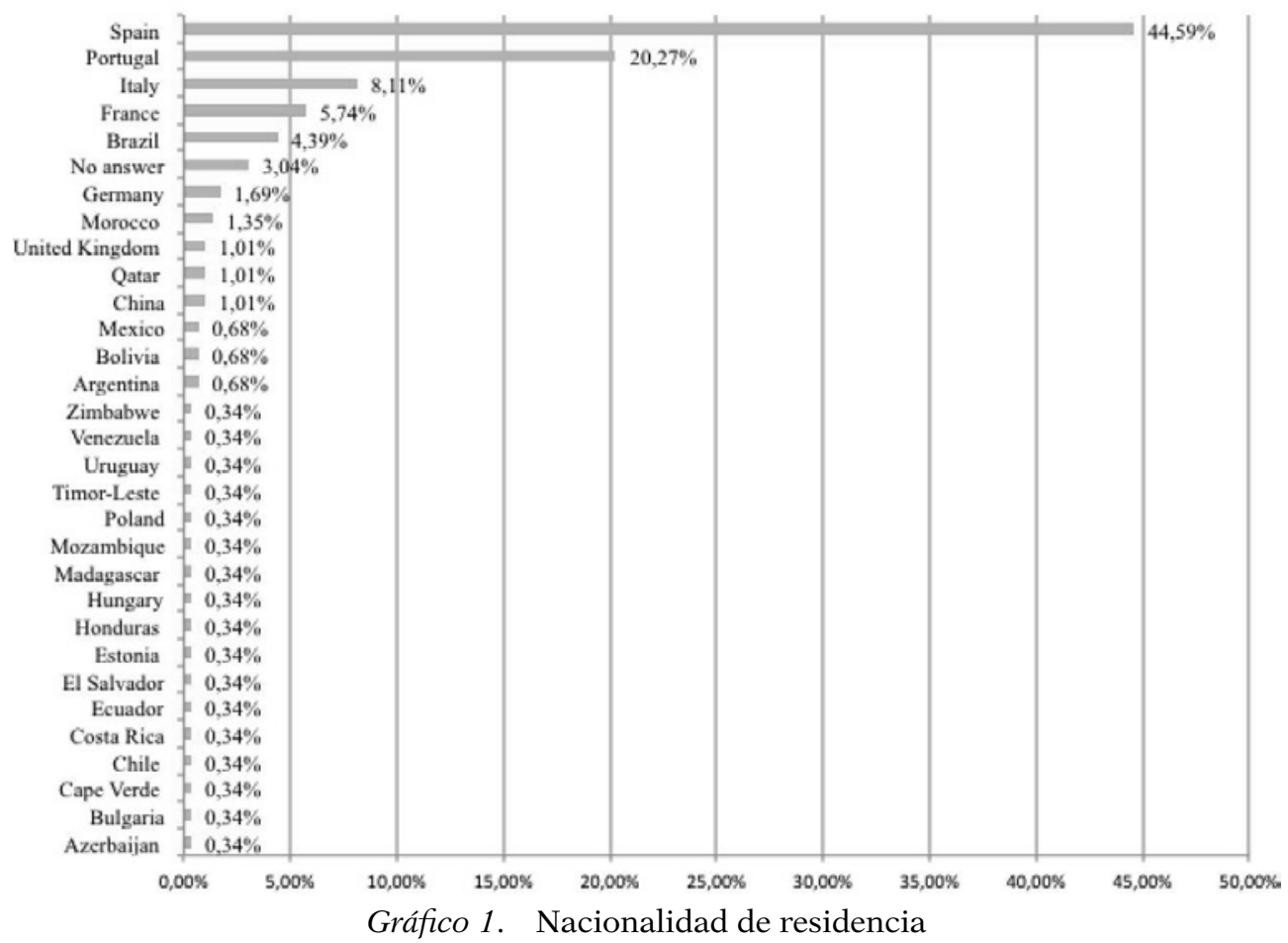

Fuente: Fueyo, A. et al. (2015) 
Esta masividad se ha visto reforzada por la formación de los participantes que, coincidiendo con otros estudios (Sánchez Acosta, 2013) y corroborando otra de las hipótesis de nuestro estudio, observamos que la mayoría tienen conocimientos suficientes para terminar el curso con éxito, tratándose de doctores, graduados o licenciados universitarios $(70,61 \%)$, cuya finalidad a la hora de elegir este tipo de formación es aumentar su especialización para su ámbito laboral, como queda reflejado en el Gráfico 2. Estos datos contrastan con los señalados en otros estudios (Ramírez, 2013; García, Tenorio \& Ramírez, 2015), que presentan como un impedimento para la participación óptima en el curso, la falta de formación previa a nivel de conocimiento instrumental de las herramientas digitales. Dentro de las distintas realidades presentes a nivel académico y profesional, la mayoría de los miembros de la comunidad de aprendizaje de los MOOC de ECO pertenece a disciplinas relacionadas con la educación $(51,04 \%)$, finalidad que persiguió este proyecto desde sus inicios y que, como podemos analizar en el Gráfico 3, está siendo alcanzada.

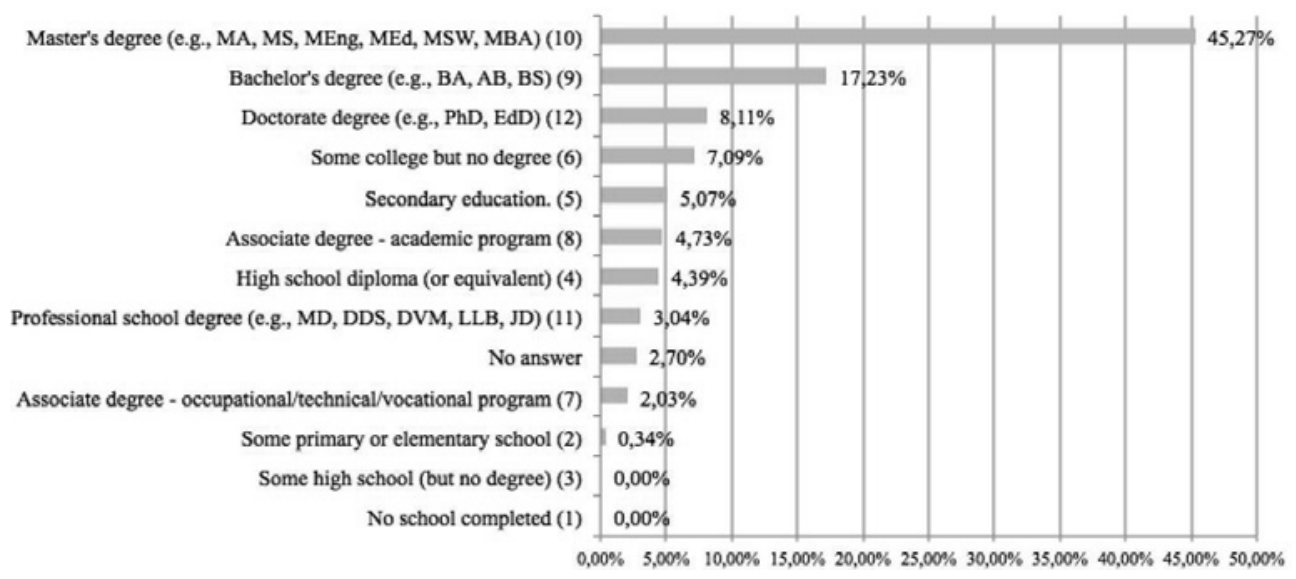

Gráfico 2. Nivel académico de los participantes

Fuente: Fueyo, A. et al. (2015) 


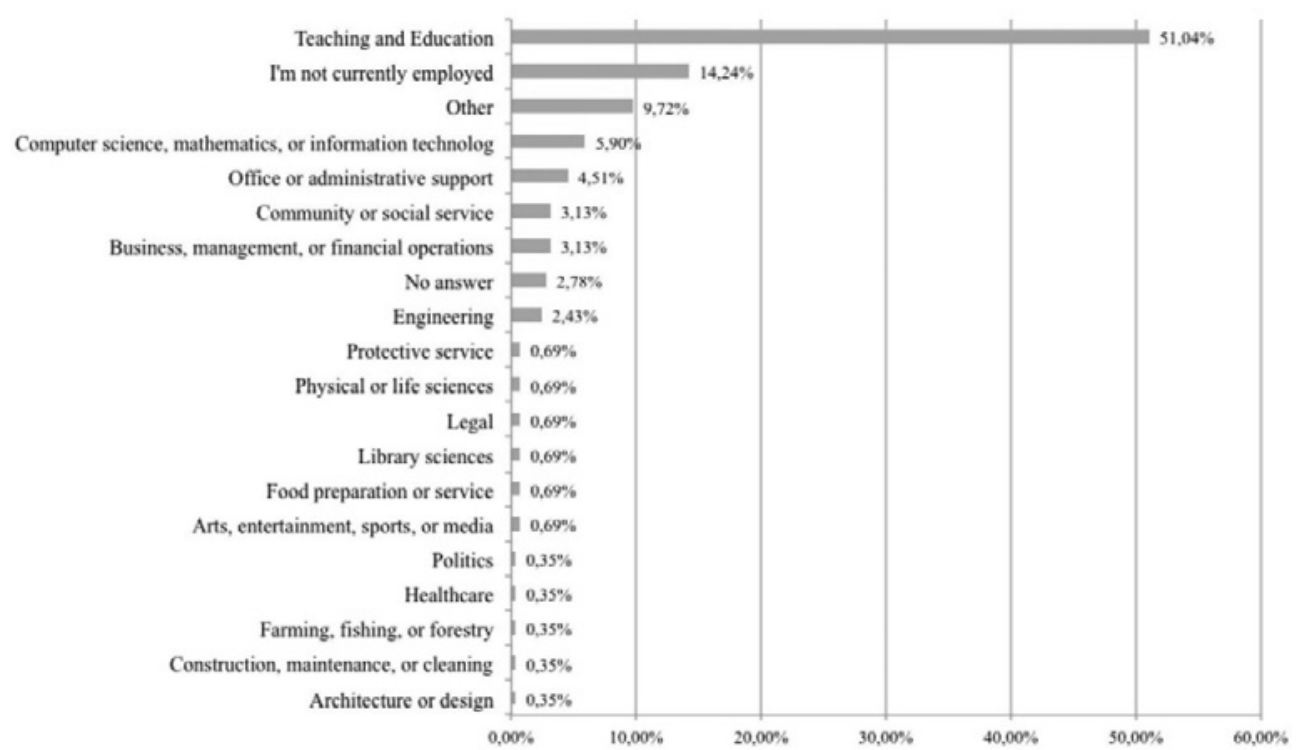

Gráfico 3. Ámbito de estudio o situación laboral

Fuente: Fueyo, A. et al. (2015)

La experiencia vivida en esta modalidad de formación potencia la necesidad de los sujetos participantes por compartir con el entorno social los beneficios de cursar MOOC. Por este motivo la muestra, como también presenta el Gráfico 4, nos ha descubierto que el carácter online de estos cursos se refleja también en el acceso a la información sobre los mismos, quedando demostrado que los espacios web $(43,5 \%)$ y el software social $(27,5 \%)$ se convierten en los canales que mejor han ayudado en la divulgación de la oferta formativa que el Proyecto ECO ha presentado a través de su plataforma. En este sentido, no solo se corrobora la primera hipótesis, como hemos indicado anteriormente, sino que además observamos que los propios participantes $(27,5 \%)$ de los MOOC se convierten en publicistas de estos cursos a través de sus perfiles virtuales. Partiendo de esta realidad, observamos que es fundamental apostar por ofrecer una buena calidad formativa a través de MOOC, fomentando el desarrollo de comunidades virtuales de aprendizaje proyectadas hacia campos de práctica, donde se vivencie la formación recibida y se impulse el interés por despertar esta misma necesidad a las comunidades que cada individuo estructura y dinamiza desde sus propios social media. 


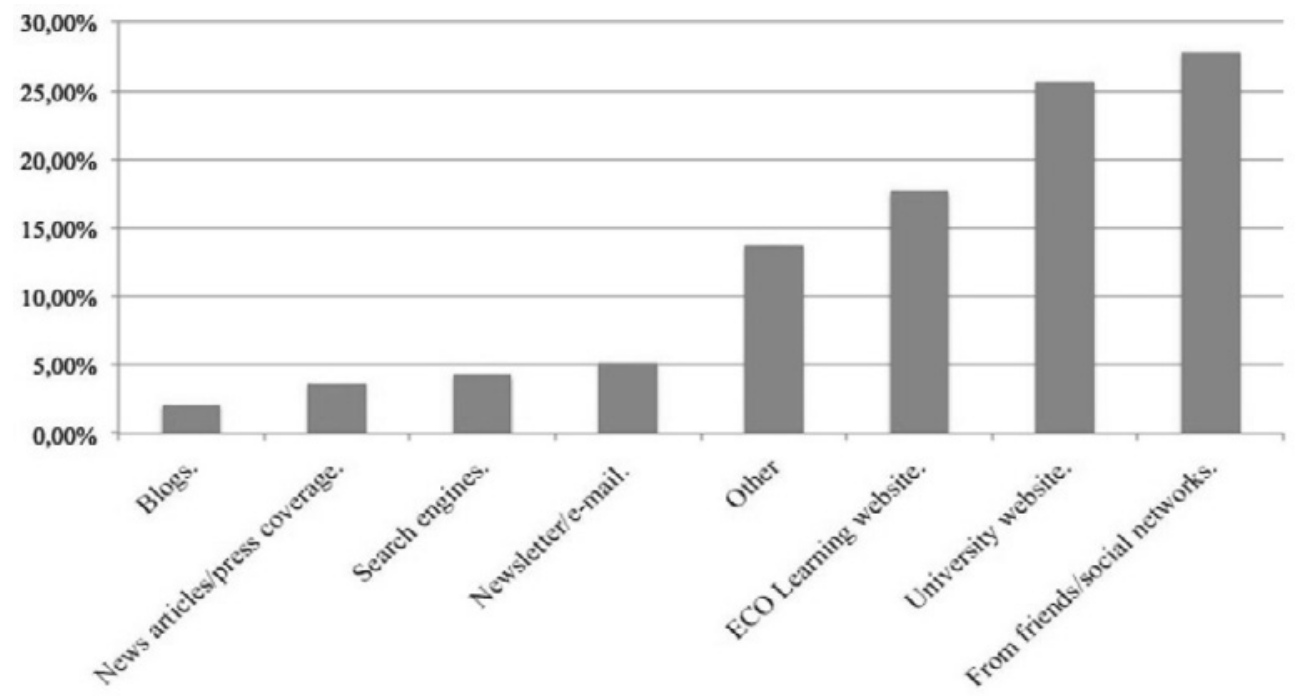

Gráfico 4. Espacios donde se ha descubierto la formación con MOOC

Fuente: Fueyo, A. et al. (2015)

Este interés por publicitar los MOOC, en los que el alumnado ha participado, no sería una realidad si no hubiese partido de una experiencia satisfactoria en la realización del curso. Los datos obtenidos que se reflejan en el Gráfico 5 muestran que las personas que han formado la comunidad virtual de aprendizaje han obtenido una grata satisfacción con la formación recibida $(85 \%)$ corroborando de esta forma la última hipótesis de nuestro estudio. Esta realidad es fruto del interés del equipo docente y de los facilitadores por desarrollar una propuesta pedagógica interactiva y un modelo comunicativo horizontal y bidireccional, coincidiendo estos resultados con los obtenidos por otras investigaciones (Mackness, Mak \& Willians, 2010; Gallego-Arrufat \& Gutiérrez-Santiuste, 2015; Gallego-Arrufat, Gámiz Sánchez \& Gutiérrez-Santiuste, 2015). Este esfuerzo por convertir los MOOC en espacios de participación ha posibilitado que el alumnado se haya sentido integrado como «un protagonista más» dentro de la comunidad, compartiendo esta experiencia e informando de los cursos de este Proyecto con el mismo interés que si fueran propios. 


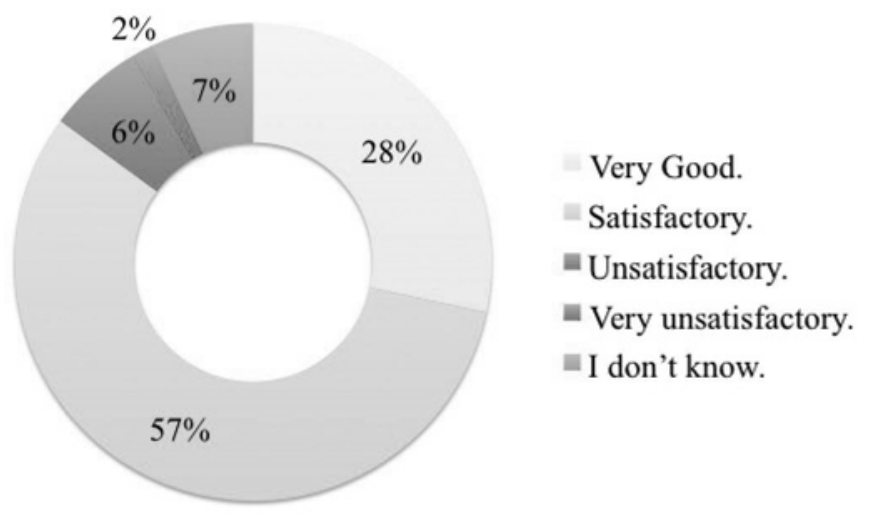

Gráfico 5. Valoración de la experiencia general del MOOC

Fuente: Fueyo, A. et al. (2015)

Tradicionalmente se han relacionado los MOOC con la alta tasa de abandono de los estudiantes y su baja participación (Belenger \& Thornton, 2013) señalando que, en general, es muy bajo el porcentaje del alumnado que sigue en realidad el curso y participa, de una forma u otra, en el mismo. A pesar de estos datos que podemos valorar, se va observando una progresiva disminución de las tasas de abandono (Cabero, Llorente \& Vázquez, 2014) y un aumento de interés por este modelo de formación. No podemos olvidar que en los MOOC nos encontramos con todo tipo de alumnado que cumple roles de participantes como observadores, merodeadores, estudiantes pasivos o más activos (Hill, 2012), que nos lleva a reconocer que estas tipologías influyen en el desarrollo del proceso formativo caracterizado por la apertura y masividad de los MOOC.

Los datos recogidos en la muestra reflejan que los participantes en los MOOC manifiestan un nivel alto de satisfacción con la formación recibida, según podremos observar a continuación, junto al resto de los gráficos, en el Gráfico 6 acerca del cumplimiento de los objetivos del curso (81\%). Al tratarse de una propuesta formativa abierta, podemos observar que no todo el alumnado que ha participado ha finalizado el MOOC. Sin embargo, se están consiguiendo números superiores de participantes que terminan sus estudios comparándolos con MOOC impartidos en otras plataformas y por otras instituciones y estudios señalados anteriormente. Asimismo, se valora de forma muy positiva cómo el alumnado que, por diferentes motivos, no ha podido acabar los contenidos que el MOOC requería para ser considerado como finalizado, sí manifiesta interés en volver a matricularse en la siguiente convocatoria ( $35 \%$ ) para intentar completar lo que en la actual no hicieron, como queda mostrado en el alto porcentaje recogido en el Gráfico 7, mos- 
trando una gran satisfacción por la calidad de los materiales audiovisuales presentados $(67 \%)$ como refleja el Gráfico 8 y por los trabajos propuestos (65\%) como recoge el Gráfico 9. Seguramente, sean estos, calidad de materiales y actividades, las principales claves para alcanzar un adecuado nivel de satisfacción de quienes se acercan a la realidad MOOC. En otro sentido, observamos también en el Gráfico 10 cómo los participantes $(78 \%)$ tienen interés por seguir cursando otros MOOC del Proyecto ECO, con lo que se está validando de alguna forma el modelo formativo que está implícito en todos los cursos ofertados. Los gráficos anteriormente explicados se pueden observar a continuación:

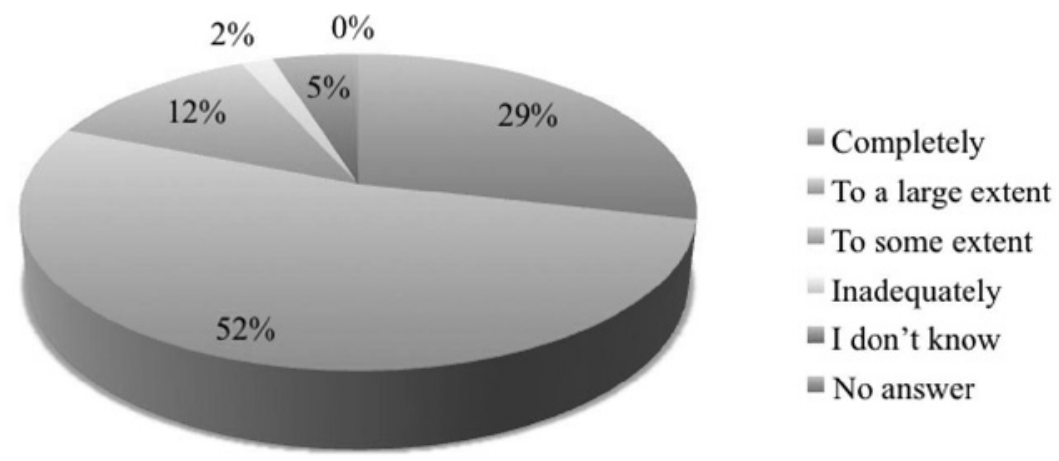

Gráfico 6. Cumplimiento de los objetivos del curso

Fuente: Fueyo, A. et al. (2015)

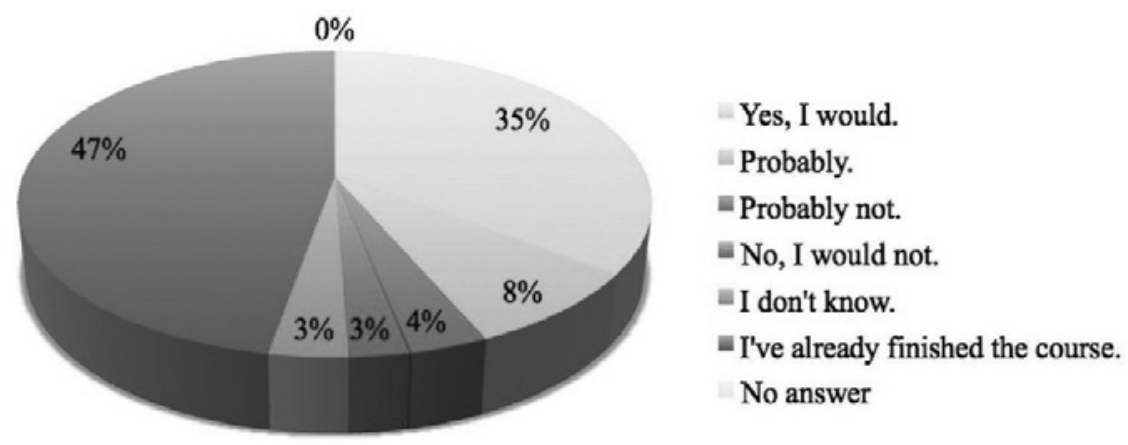

Gráfico 7. Intencionalidad de terminar el curso en otras convocatorias

Fuente: Fueyo, A. et al. (2015) 


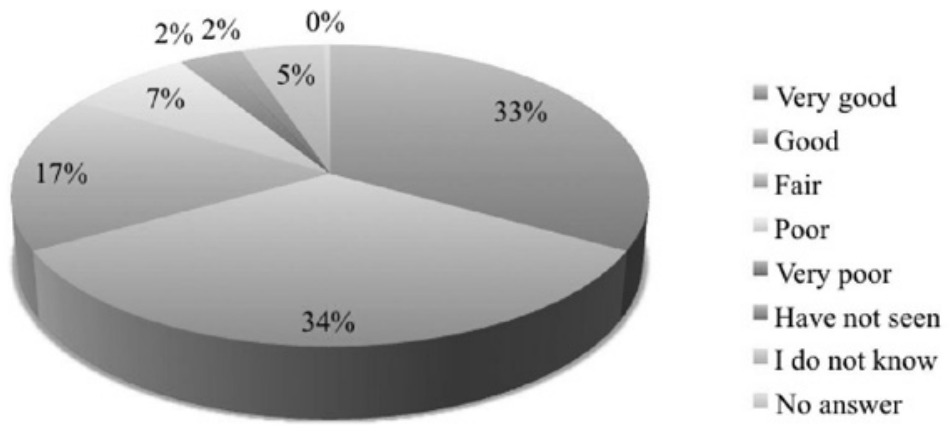

Gráfico 8. Satisfacción sobre los materiales audiovisuales ofrecidos en el curso Fuente: Fueyo, A. et al. (2015)

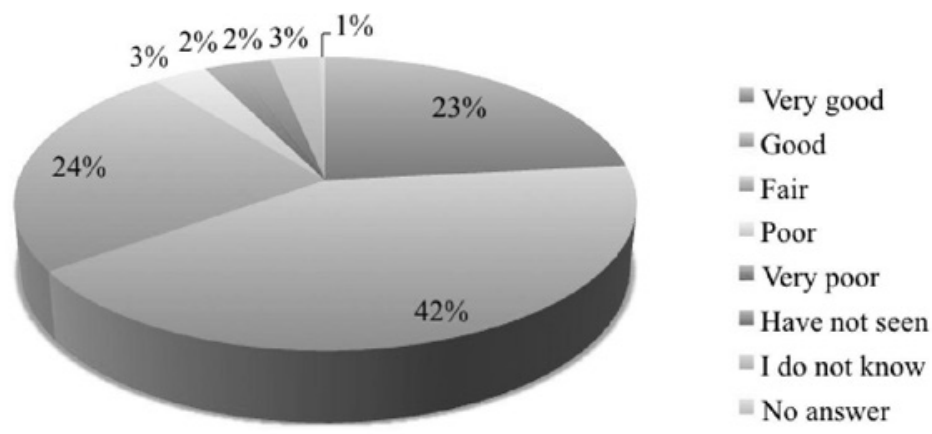

Gráfico 9. Satisfacción por el planteamiento de los trabajos solicitados en el curso Fuente: Fueyo, A. et al. (2015)

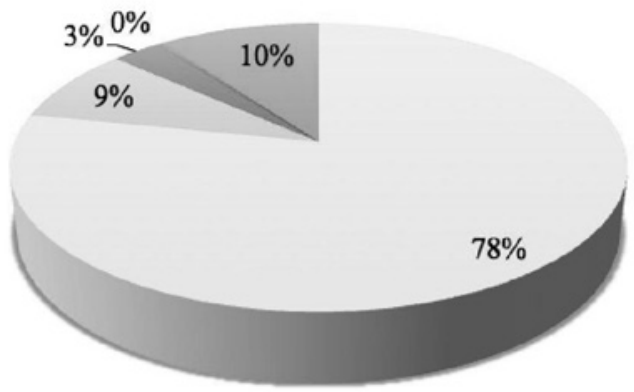

\author{
Yes, I would. \\ Yes, I would, but in a different area \\ of knowledge. \\ = Probably not. \\ = No, I would not. \\ I I don't know.
}

Gráfico 10. Intencionalidad de realizar otro MOOC del Proyecto europeo ECO Fuente: Fueyo, A. et al. (2015)

Para concluir con los resultados de nuestra investigación debemos recordar, coincidiendo con otros estudios (Gil-Quintana, 2016), que una de las apuestas más fuertes por las que ha optado el Proyecto ECO ha sido el 
acceso a estos cursos para cualquier persona, independientemente de sus características, poniendo el acento en la apertura de los MOOC; no solo en el diseño accesible y usabilidad de la plataforma, sino a la respuesta interactiva para corresponder a la demanda social por un nuevo estilo de aprendizaje, que prioriza el hecho de poner al alumnado en el centro de la acción educativa y por el que han apostado otros autores como Chatti (2010). Desde esta perspectiva observamos cómo la muestra representada en los Gráficos 11 y 12 del estudio cuantitativo ha evaluado este aspecto de usabilidad $(56 \%)$ y accesibilidad $(72 \%)$ otorgando una valoración muy positiva a este criterio.

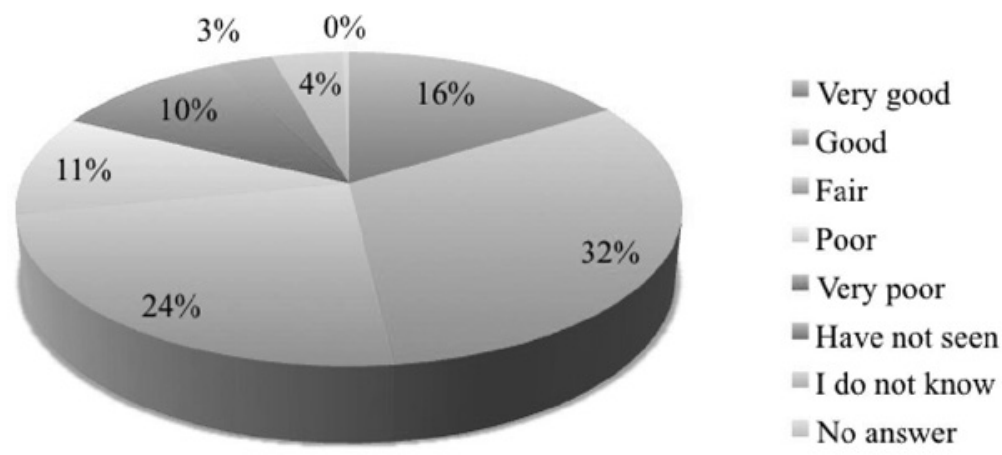

Gráfico 11. Satisfacción acerca de la usabilidad de la plataforma

Fuente: Fueyo, A. et al. (2015)

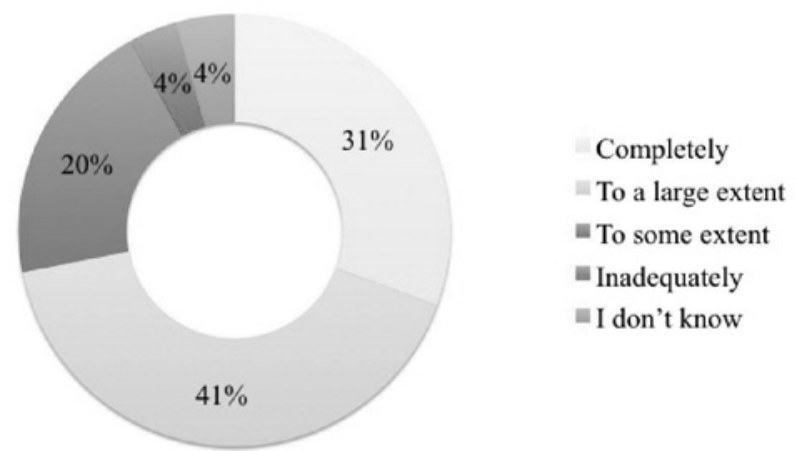

Gráfico 12. Los contenidos MOOC accesibles para personas con distintas habilidades de aprendizaje

Fuente: Fueyo, A. et al. (2015)

No obstante, observamos que son muy escasos los participantes que acceden a los MOOC de ECO con algún tipo de discapacidad física, sensorial o intelectual que hayan podido validar completamente la accesibilidad de los cursos. Realmente, faltaría atraer a estos participantes en próximas ediciones. 


\section{CONCLUSIONES}

La nueva realidad MOOC está proyectándose hacia un modelo formativo con una identidad propia. Este ámbito está creciendo tan rápidamente que lo consideramos de vital importancia, siendo signo visible de una nueva realidad digna de ser estudiada e investigada desde nuevas y futuras perspectivas. Aportando desde la investigación a este campo de estudio procedemos a exponer las diferentes conclusiones principales que derivan de nuestra investigación, basándonos en los objetivos que han guiado el estudio y que han servido de base para el desarrollo metodológico.

- El Proyecto europeo ECO ha potenciado el carácter masivo de los MOOC haciendo presente esta formación, no solo en países europeos, sino también en otros continentes y, aunque de forma más reducida, en países africanos. La convivencia de distintas culturas e idiomas en una comunidad de aprendizaje masiva como la de ECO es uno de los factores que más enriquecen la calidad de los cursos ofrecidos desde el propio proyecto.

- Los participantes en los MOOC del Proyecto europeo ECO tienen un nivel formativo propio de graduados, máster o doctorado y su principal dedicación es la docencia. En este sentido, su predisposición para la realización del curso es eficiente y su motivación versa principalmente por el enriquecimiento de su currículum para una mayor especialización o formación en un determinado ámbito profesional. La pretensión última es que estos docentes creen sus propios MOOC dentro del proyecto y multipliquen, de esta forma, el radio de acción de los cursos masivos a otros perfiles formativos de la ciudadanía europea, especialmente a la infancia, adolescencia y jóvenes.

- El acceso a la información sobre esta modalidad formativa o el descubrimiento de estos cursos por parte de los participantes ha sido a través de diferentes escenarios digitales, en las redes sociales o en la misma plataforma del Proyecto europeo ECO. De forma general, el alumnado se ha convertido en publicista de esta plataforma otorgando mayor credibilidad en su criterio, al partir de su propia experiencia positiva en la misma.

- La experiencia de los participantes en los MOOC del Proyecto europeo ECO se valora como muy satisfactoria, mostrando interés en seguir cursando estas propuestas de formación en convocatorias sucesivas o en otras temáticas dentro de la misma convocatoria. 
- Los documentos audiovisuales presentados y los trabajos requeridos por parte del alumnado que cursa los MOOC del Proyecto europeo ECO son evaluados de forma satisfactoria. Son Recursos Educativos Abiertos (REA) que se presentan inicialmente en los cursos a través de documentos en formato textual y en formato multimedia. Su característica principal es que los participantes reutilizan y recrean dichos REA, convirtiéndose en coautores de los mismos dentro las «comunidades tecnosociales» (Camarero-Cano, 2015) proyectadas desde los MOOC.

- La apertura ha sido otra de las características de los MOOC del Proyecto europeo ECO al fomentar la usabilidad y ofrecer la posibilidad de acceso a cualquier persona. La dificultad en el acceso a los contenidos en abierto debido a diferentes discapacidades o dificultades en el aprendizaje no se ha dado en estos cursos, otorgando al carácter de abierto un mayor significado. El objetivo es llevar a cabo en los MOOC el «diseño universal» o «diseño para todos» con el fin de no dejar a nadie fuera de este tipo de formación masiva y online.

Finalmente, es importante destacar que aunque algunas investigaciones (Luo \& Robinson, 2014) destacan la gran satisfacción de los estudiantes ante el tipo de evaluación por pares (P2P) llevado a cabo en los MOOC, las reflexiones realizadas al respecto por los estudiantes de los cursos de ECO presentan una gran dicotomía. Por un lado, coincide la gran satisfacción con la evaluación P2P, que nos remite a la idea del empoderamiento de los estudiantes como máxima de ECO, y, por otro, el rechazo de la misma, prefiriendo en este último caso que fueran los docentes quienes llevaran a cabo la labor de evaluación y no sus propias compañeras y compañeros.

Se hace patente que los desafíos más importantes a los que se enfrenta la universidad, por la proyección de estos nuevos espacios de formación, derivan de las innovaciones disruptivas que llevan consigo. Dichos desafíos ocasionan cambios en los paradigmas y roles educativos, que elevan la construcción colectiva del conocimiento como un valor social, repercutiendo positivamente en el desarrollo de la ciudadanía digital. De ello se desprende la necesidad de consolidar un modelo comunicativo bidireccional y horizontal, además de una pedagogía interactiva y participativa, que promuevan el empoderamiento del alumnado para su repercusión en la mejora de la capa social. A este reto se añaden nuevas vías de investigación, como es el estudio y desarrollo de herramientas comunicativas que ayuden a convertir las aulas digitales en escenarios educomunicativos y responder así a las demandas que actualmente realizan los contextos sociales en que están enclavadas. 
Los MOOC son una ventana abierta al cambio social que puede ser potencializada por nuestras propias manos. Dada la incertidumbre que rodea este fenómeno y su carácter prematuro, no parece que tenga mucho sentido el tratar de realizar conclusiones cerradas o predicciones futuristas sobre este modelo formativo. No obstante, hemos pretendido aportar un "granito de arena» a esta nueva realidad que, sin duda alguna, está rompiendo los esquemas del aprendizaje en las instituciones universitarias. 


\section{REFERENCIAS BIBLIOGRÁFICAS}

Alcina Franch, J. (1999). Aprender a investigar. Madrid: Compañía Literaria.

Barbas, A. (2012). Educomunicación: desarrollo, enfoques y desafíos en un mundo interconectado. Foro de Educación, $n .^{\circ} 14$, pp. 157-175.

Belenger, S. \& Thornton, J. (2013). Biolectricity: A Quantitative Approach. Duke's University's First MOOC. Recuperado de: https://goo.Gl/yniun

Cabero Almenara, J., Llorente Cejudo, M. C., y Vázquez Martínez, A. I. (2014). Las tipologías de MOOC: su diseño e implicaciones educativas. Profesorado. Revista de currículum y formación del profesorado. Recuperado de: https://goo.gl/cbO8Jb

Camarero-Cano, L. (2015). Comunidades tecnosociales. Evolución de la comunicación analógica hacia la interacción analógico-digital. Revista Mediterránea de Comunicación, vol. 6(1), 187-195. DOI 10.14198/MEDCOM2015.6.1.11.

Cantillo, C. (2014). Nuevas dinámicas de aprendizaje en entornos virtuales. En Osuna, S. (coord.): Escenarios virtuales educomunicativos. Barcelona: Icaria.

Chatti, M. A. (2010). Personalization in Technology Enhanced Learning: A Social Software Perspective. RWTH Aachen University, Shaker Verlag.

Coller, X. (2005). Estudio de casos. Cuadernos metodológicos, n. ${ }^{\circ}$ 30. Madrid: Centro de Investigaciones Sociológicas.

Cormier, D. (2010). What is a MOOC). [Youtube]. Recuperado de: https://goo. $\mathrm{gl} /$ tnpqrl

Curley, N. (2014). Online education platform Coursera blocks students in Syria and Iran. [Wamda]. Recuperado de: http:// https://goo.gl/felNU7
Downes, S. (2013). MOOC-The Resurgence is Community in Online Learning. Recuperado de: http://goo.gl/1GPk3y

Freire, P. (1975). Pedagogía del oprimido. Madrid: Siglo XXI.

Freire, P. (2002). La educación como práctica de la libertad. Madrid: Siglo XXI.

Fueyo, A. et al. (2015). ECO_D4.3 Report on users satisfaction year 1. Recuperado de: https://goo.gl/YurDdS

Gallego-Arrufat, M. J., Gámiz Sánchez, V. y Gutiérrez-Santiuste, E. (2015). Tendencias en la evaluación del aprendizaje en cursos en línea masivos y abiertos. Educación XX1, 18(2), 77-96. DOI: $10.5944 /$ educXX1.12935.

García, B. J., Tenorio, G. C. \& Ramírez, M. S. (2015). Retos de automotivación para el involucramiento de estudiantes en el movimiento educativo abierto con MOOC. RUSC. Universities and Knowledge Society Journal, 12(1), 91104. DOI: 10.7238/rusc.v12i1.2185.

Gil-Quintana, J. (2015). MOOC «Innovación Educativa y desarrollo profesional. Posibilidades y límites de las TIC». Una experiencia desde la educomunicación en el Proyecto europeo ECO. Qualitative Research in Education, 4(3), 299-328. DOI:10.17583/ qre.2015.1518.

Gil-Quintana, J. (2016). La nueva realidad sMOOC, una ventana abierta a la educomunicación. Revista Mediterránea de Comunicación, 7 (2). DOI: 10.14198/MEDCOM2016.7.2.5.

Hill, P. (2012). Four Barriers that MOOCs must overcome to build a sustainable model. e-Literate. Recuperado de: https://goo.gl/7F9Rs 
López-Meneses, E. Vázquez-Cano, E. y Román, P. (2014). Análisis e implicaciones del movimiento MOOC en la comunidad científica: JCR y SCOPUS. Revista Científica de Comunicación y Educación: Comunicar. DOI: 10.3916/ C44-2015-08.

Luo, H. y Robinson, A. C. (2014). Is peer grading a valid assessment method for Massive Open Online Courses (MOOC)? 7th Annual International Symposium. Emerging Technologies for Online Learning. Recuperado de: https://goo.gl/7rgOeQ

Luque (2015). Principales tendencias y desafíos para el aprendizaje en línea. América Learning \& Media. Recuperado de: https:/goo.gl/E0dE6V

Mackness, J., Mak, S. \& Williams, R. (2010). The ideals and reality of participating in a MOOC. In L. DirckinckHolmfeld, V. Hodgson \& Al. (Eds.). Proceedings of the 7th International Conference on Networked Learning (pp. 266-275). Lancaster: University of Lancaster.

Martín, F. G. (2012). Will Massive Open Online Courses Change how we Teach? Communications of the ACM, 55 (8), 26-28. Revista Científica de Comunicación y Educación: Comunicar. DOI: 10.1145/2240236.2240246.

Osuna-Acedo, S. (2014). La web 2.0 y la educación a lo largo de la vida. En Aranda, D.; Creus, A.; Sánchez-Navarro, J.: Educación, medios digitales y cultura de la participación. Barcelona: Editorial UOC.

Osuna-Acedo, S. y Aparici, R. (2014). Escenarios virtuales para una sociedad del conocimiento. Osuna, S. (coord.): Escenarios virtuales educomunicativos. Barcelona: Icaria.
Osuna-Acedo, S. (cord.) (2014-2017). ECO Project (Elearning, Communication, Open-Data). Recuperado de: http://project.ecolearning.eu

Pernías, P. y Luján, S. (2014). Los MOOC: orígenes, historia y tipos. Recuperado de: https://goo.gl/8BVzhG

Patru, M. \& Balaji, V (editors) (2016). Making sense of MOOCs: A guide for policy-makers in developing countries. France and Canada: UNESCO and Commonwealth of Learning.

Ramírez, M. (2013). Competencias Docentes y Prácticas Educativas Abiertas en la Educación a Distancia. Monterrey: LULU editorial digital. Recuperado de: https://goo.gl/y8Yp1m

Raposo-Rivas, M. Martínez-Figueira, E. y Sarmiento, J. A. (2014). Un estudio sobre los componentes pedagógicos de los cursos online masivos. Revista Científica de Comunicación y Educación: Comunicar. DOI: 10.3916/ C44-2015-03.

Rodríguez Terceño, J. (2012). Aplicaciones del EEES a partir de la Web 2.0 y 3.0. Madrid: Visión Libros.

Román Mendoza, E. (2014). Los medios sociales en los cursos online abiertos y masivos (MOOC): un cambio hacia la innovación a través de la disrupción y la colaboración. En Aranda, D.; Creus, A.; Sánchez-Navarro, J.: Educación, medios digitales y cultura de la participación. Barcelona: Editorial UOC.

Sánchez Acosta, E. (2013). MOOC: resultados reales. Recuperado de: https://goo.gl/OVFruz

Sancho Vinuesa, T.; Oliver, M. y Gisbert, M. (2015). MOOCS en Cataluña: Un instrumento para la innovación en educación superior. Educación $X X 1,18(2), 125-146$. DOI: 10.5944/ educXX1.13462. 
Sangrá, A. y Wheeler, S. (2013). Nuevas formas de aprendizaje informales: ¿O estamos formalizando lo informal? Revista de Universidad y Sociedad del Conocimiento. Barcelona: Universitat Oberta de Catalunya.

Siemens, G. \& al. (2012). How This Course Works. Learning Analytics and Knowledge: LAK12. Recuperado de: https://goo.gl/wIdWyv

Silva, M. (2005). Educación interactiva. Enseñanza y aprendizaje presencial y on-line. Barcelona: Gedisa.
Tobías-Martínez, M. A., Duarte-Freitas, M. C. y Kemczinski, A. (2015). Un repositorio digital de contenido fílmico como recurso didáctico. Revista Científica de Comunicación y Educación: Comunicar. Recuperado de: https:// goo.gl/GHyQzN

Vázquez Cano, E. López-Meneses, E. y Sarasola, J. L. (2013). La expansión del conocimiento en abierto: Los MOOC. Barcelona: Octaedro.

Yuan, L. \& Powell, S. (2013). MOOC and Open Education: Implications for Higher Education. Cetis. Recuperado de: https://goo.gl/OwRVd 


\section{PERFIL ACADÉMICO Y PROFESIONAL DE LOS AUTORES}

Sara Osuna-Acedo. Doctora en Filosofía y Ciencias de la Educación por la UNED. Profesora de Comunicación y Educación de los Grados de Pedagogía y de Educación Social en la UNED y especialista en tecnologías digitales, modelos educomunicativos y enseñanza virtual. Ha colaborado con universidades latinoamericanas en diferentes proyectos sobre Aprendizaje Colaborativo y Modelos Comunicativos. Sus líneas de investigación son MOOC, convergencia de medios, escenarios digitales, discapacidad, aprendizaje digital y redes sociales.

Javier Gil-Quintana. Doctor en Educación y Comunicación en la Red por la UNED. Actualmente colabora como profesor en la facultad de Educación de la UNED (Madrid) y forma parte del equipo docente de los MOOC del Proyecto Europeo ECO (UNED), colaborando también con su equipo de investigación. Esta labor investigadora se centra en los nuevos espacios formativos MOOC, presentando diferentes comunicaciones en congresos y artículos en diversas revistas de impacto.

Dirección de los autores: Sara Osuna-Acedo

Facultad de Educación (UNED)

Calle Juan del Rosal, 14. Despacho 236

28040 Madrid

E-mail: sosuna@edu.uned.es

Javier Gil-Quintana

Facultad de Educación (UNED)

Calle Juan del Rosal, 14. Despacho 236

28040 Madrid

E-mail: jgilquintana@invi.uned.es

Fecha Recepción del Artículo: 10. Enero. 2016

Fecha modificación Artículo: 26. Junio. 2016

Fecha Aceptación del Artículo: 27. Julio. 2016

Fecha Revisión para Publicación: 14. Febrero. 2017 
